\title{
Philosophiques
}

\section{Le nominalisme d'Ockham et la représentation mentale. Précis de Ockham on Concepts}

\section{Claude Panaccio}

Volume 32, numéro 2, automne 2005

URI : https://id.erudit.org/iderudit/011876ar

DOI : https://doi.org/10.7202/011876ar

Aller au sommaire du numéro

Éditeur(s)

Société de philosophie du Québec

ISSN

0316-2923 (imprimé)

1492-1391 (numérique)

Découvrir la revue

Citer ce document

Panaccio, C. (2005). Le nominalisme d'Ockham et la représentation mentale. Précis de Ockham on Concepts. Philosophiques, 32(2), 423-426.

https://doi.org/10.7202/011876ar d'utilisation que vous pouvez consulter en ligne.

https://apropos.erudit.org/fr/usagers/politique-dutilisation/ 


\title{
Disputatio
}

\section{Le nominalisme d'Ockham et la représentation mentale. Précis de Ockham on Concepts'}

\author{
CLAUDE PANACCIO \\ Université du Québec à Montréal \\ panaccio.claude@uqam.ca
}

Les études ockhamistes ont connu un essor considérable ces vingt dernières années. Deux facteurs principaux, me semble-t-il, ont joué de concert à ce propos. D'abord, une excellente édition critique des écrits philosophiques et théologiques d'Ockham, achevée en 1988, a rendu l'œuvre disponible aux chercheurs dans une version fiable, ce qui, malheureusement, reste exceptionnel en études médiévales: on n'a pas encore l'équivalent pour Abélard, Albert le Grand ou Jean Duns Scot, par exemple. Il faut en savoir gré au Franciscan Institute de l'Université Saint-Bonaventure (N.Y.), qui a consacré quarante ans à l'entreprise, et au Père Gedeon Gál, en particulier, qui l'a dirigée de main de maître pendant plus de vingt ans et qui l'a menée à terme ${ }^{2}$.

Deuxièmement, il y a eu une prise de conscience nette, ces dernières décennies, d'affinités frappantes entre la philosophie de la fin du Moyen Âge,

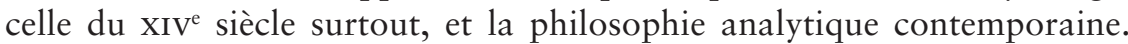
L'ouvrage qui l'a le mieux manifesté est le Cambridge History of Later Medieval Philosophy paru en 1982 sous la direction de Norman Kretzmann, Anthony Kenny et Jan Pinborg, qui a résolument mis l'accent sur l'intérêt du Moyen Âge tardif pour les discussions actuelles en philosophie analytique ${ }^{3}$. Il n'est pas abusif de dire, de fait, que la via moderna de la fin du Moyen Âge - dont Guillaume d'Ockham est l'un des grands représentants - est, littéralement, une philosophie de type analytique: une pratique philosophique, c'est-à-dire, qui repose sur des théories logiques et sémantiques détaillées et sophistiquées, et qui utilise systématiquement ces appareils logico-sémantiques pour la discussion de tous les problèmes philosophiques. Les questions

1. Panaccio, Claude, Ockham on Concepts, Aldershot, G.-B., Ashgate, 2004, xi + $197 \mathrm{p}$.

2. L'édition des Opera philosophica et theologica d'Ockham par le Franciscan Institute de l'Université Saint-Bonaventure (N.Y.) est répartie en deux séries: Opera philosophica, éd. par G. Gál et al., 7 volumes, 1974-1988, et Opera theologica, éd. par G. Gál et al., 10 volumes, 19671986. L'édition des œuvres politiques, en revanche, qui est indépendamment menée, est encore incomplète: Opera politica, éd. par H. S. Offler et al., 4 volumes (jusqu’à présent): volumes I-III, Manchester, Manchester University Press, 1940-1963; volume IV, Oxford, Oxford University Press, 1997.

3. Cambridge History of Later Medieval Philosophy, sous la dir. de N. Kretzmann, A. Kenny et J. Pinborg, Cambridge, Cambridge University Press, 1982. 
de philosophie de la physique, par exemple, sont en grande partie, chez Ockham, des problèmes de sémantique des termes comme "mouvement", «vide», «lieu », «temps », etc.

La mise à disposition des textes mêmes d'Ockham, donc, et la prise de conscience de l'intérêt potentiel de ces textes pour les discussions en cours en philosophie du langage, en philosophie de l'esprit, en métaphysique analytique, etc., ont concouru à susciter de nombreux travaux sur Ockham depuis une vingtaine d'années, à commencer par l'exceptionnel William Ockham de Marilyn Adams en $1987^{4}$. Et ce foisonnement de recherches a donné lieu, comme de raison, à d'importants débats d'interprétation au sujet de la doctrine ockhamiste. Il se trouve que les plus agités de ces débats, depuis 1990 surtout, ont tourné autour de la théorie des concepts. J'y ai contribué, comme plusieurs autres. Et j'ai senti le besoin, à un certain moment, de faire le point là-dessus d'une manière systématique. Tel est l'objet de ce livre, que j'ai fait en anglais pour rejoindre directement mes interlocuteurs anglophones, sans être inaccessible aux autres pour autant.

Les trois premiers chapitres couvrent les notions de base de la théorie ockhamiste des concepts:

- la distinction entre connaissance intuitive et connaissance abstractive, et l'idée générale de langage mental (au chapitre 1);

- l'idée que les concepts sont des actes intellectuels (au chapitre 2);

- l'idée que les concepts sont des signes (au chapitre 3).

J'ai essayé, chaque fois, de mener l'exposition en discussion avec les travaux récents - ceux, en particulier, avec lesquels je me trouve en désaccord. Mais j'ai voulu surtout que les enjeux en soient compréhensibles au noninitié.

Les trois chapitres suivants abordent un thème qui est devenu central dans les discussions actuelles sur Ockham, celui des concepts connotatifs. Un terme connotatif, chez Ockham, est une unité signifiante dotée d'une structure sémantique hiérarchisée. Il a des signifiés premiers d'une part - une extension, si l'on préfère: "blanc", par exemple, a pour signifiés premiers toutes les choses blanches, et «cavalier» a pour signifiés premiers tous les cavaliers. Mais le terme connotatif, outre son extension, a aussi une référence oblique: il renvoie à d'autres individus du monde, qui ne font pas, d'habitude, partie de son extension. C'est là sa connotation. Ainsi, «cavalier» évoque les chevaux, "blanc» les blancheurs (Ockham admet que chaque chose blanche possède sa propre blancheur, qui est une qualité singulière), "père » encore - un des exemples favoris d'Ockham - évoque les enfants, tous ces connotata n'étant jamais pour Ockham que des entités singulières, au même titre que les signifiés premiers.

4. Adams, Marilyn M., William Ockham, 2 volumes, Notre Dame, Ind., University of Notre Dame Press, 1987. 
Le problème d'interprétation qui s'est posé au sujet des connotatifs chez Ockham est le suivant: y a-t-il selon lui des concepts connotatifs simples dans le langage mental qui sert aux humains à penser? La réponse dominante dans les années soixante-dix et quatre-vingt était que non. Les connotatifs, disaiton, sont représentés dans l'esprit, selon la théorie d'Ockham, par des définitions. Les connotatifs de notre langage oral, en d'autres mots, comme "père ", "blanc", ou "cavalier», ne sont, dans cette optique, que des abréviations conventionnelles pour des définitions complexes. Telle était, notamment, la lecture de Paul Vincent Spade, de Marilyn Adams et de Calvin Normore entre autres $^{5}$.

Le corollaire de cette interprétation est que les définitions complètes dans l'esprit ne doivent pas ultimement contenir elles-mêmes de connotatifs. Le programme nominaliste d'Ockham, dès lors, a été compris comme un programme d'élimination des connotatifs, dans le but de retrouver un langage sémantiquement idéal (à la Frege-Russell), mais qui corresponde en même temps au langage mental naturel dont les humains sont dotés, celui qui fournit à toutes les langues conventionnelles leur véritable ossature sémantique. Ce langage idéal serait entièrement dénué de connotatifs, et ne contiendrait que des termes "absolus» au sens d'Ockham - c'est-à-dire en gros des termes d'espèce naturelle, dépourvus de connotata. Comme les termes relationnels sont des connotatifs pour Ockham, ce programme réductionniste aurait exigé l'élimination théorique de tous les termes relationnels, sans que la capacité expressive de notre langage en soit affectée. La conséquence, évidemment, était que le programme en question était voué à l'échec: le nominalisme d'Ockham apparaissait comme une impasse.

J'ai essayé de montrer, pour ma part, dans un certain nombre de publications à partir de 1990, que tel n'était pas le programme d'Ockham6. Il accepte sans problème des concepts connotatifs dans l'esprit, qui n'y sont pas représentés par leurs définitions et qu'il ne s'agit aucunement de réduire ou d'éliminer.

5. Voir en particulier: Spade, Paul Vincent, "Ockham's distinctions between absolute and connotative terms ", Vivarium, 13, 1975, p. 55-76 (repris dans Spade, P. V., Lies, Language and Logic in the Late Middle Ages, Londres, Variorum reprints, 1988); Adams, Marilyn M., op. cit., p. 319-327; Normore, Calvin, "Ockham on mental language», dans Historical Foundations of Cognitive Science, sous la dir. de J. C. Smith, Dordrecht, Kluwer, 1990, p. 5370 ; et Spade, P. V., «Three versions of Ockham's reductionist program ", Franciscan Studies, 56, 1998, p. 347-358.

6. Panaccio, Claude, Connotative Terms in Ockham's Mental Language, Cahiers d'épistémologie, n 9016, Montréal, UQÀM, 1990, 22 p. (repris en version française avec quelques ajouts dans: Panaccio, Claude, "Guillaume d'Ockham, les connotatifs et le langage mental ", Documenti e studi sulla tradizione filosofica medievale, 51, 2000, p. 297-316); "Semantics and mental language", dans The Cambridge Companion to Ockham, sous la dir. de P. V. Spade, Cambridge, Cambridge University Press, 1999, p. 53-75; "Connotative concepts and their definition in Ockham's nominalism", dans La tradition médiévale des Catégories (xiie-xve siècles), sous la dir. de J. Biard et I. Rosier-Catach, Louvain, Peeters, 2003, p. 141-155. 
Quand on prend acte de ce point d'apparence technique, toute la perspective, progressivement, change quant au rôle que Guillaume attribue au langage mental. Ce n'est plus, en particulier, un langage logiquement idéal, il s'y trouve des ambiguités et des redondances. La conception des définitions, aussi, doit être revue, ainsi que la gnoséologie: comment acquiert-on, pour Ockham, ces concepts connotatifs élémentaires qui nous sont si utiles? C'est tout cela que j'ai essayé d'expliquer en détail au chapitre 4 (qui reprend mon texte de 1990 sur la question, avec quelques amendements), 5 (sur la théorie des définitions) et 6 (sur le processus d'acquisition des connotatifs simples).

Un dernier trio de chapitres, enfin, aborde quelques problèmes intéressants qui se posent encore au sujet de la théorie ockhamiste des concepts:

- en quel sens au juste les concepts sont-ils des similitudes des choses? (au chapitre 7);

- quel est le statut des concepts logiques, ceux que Guillaume appelle les syncatégorèmes? (au chapitre 8);

- comment les mots oraux reçoivent-ils leur signification? (au chapitre 9).

J'ai essayé de montrer notamment dans ce dernier chapitre que la position d'Ockham est résolument externaliste eu égard à la signification des mots: la signification des mots que j'utilise dépend non pas de ce que j'ai dans la tête au moment de l'énonciation, mais de ce que l' "impositeur" (l'imponens) a décrété quand le mot a été institué, décision dont je peux très bien n'avoir qu'une connaissance assez floue, ou même carrément erronée parfois. Ockham aurait souscrit à l'adage de Putnam: «meanings just ain't in the head!». Irait-il encore plus loin et souscrirait-il aussi à quelque forme d'externalisme eu égard au contenu même de nos pensées, à la signification non plus des mots oraux seulement, mais de nos concepts mêmes, compris comme des signes naturels? Je le crois en effet. Et certains éléments dans le livre le suggèrent fortement (au chapitre 7 surtout). C'est ce thème, principalement, que j'essaie d'approfondir depuis la publication du livre. 